\title{
Salience and the Cognitive Mediation of Attribution
}

\author{
Eliot R. Smith \\ Department of Sociology \\ University of California, Riverside
}

\author{
Frederick D. Miller \\ New York University
}

Two studies of the impact of salience and informational factors on attribution and memory yielded findings on two general issues. First, manipulations of the amount of thought subjects gave to their attributions and of a delay before responding to attribution questions did not diminish the effect of salience on attribution; in fact, the delay increased the effect. Second, recall of the stimulus material was shown to be influenced by salience and by covariation information (consensus, distinctiveness, and consistency) and to be related to attributions. These findings, together with theory and data from the literature on comprehension and representation of linguistic material in memory, are used to argue that salience is not simply a process by which people make attributions without giving much thought to them. Instead, salience effects reflect the close relationships among the processes of comprehension, remembering, and attribution, and the fact that attributional processing can take place at the time of the encoding and storage of information, as well as at the time of its retrieval from memory.

The literature on the perception of causation currently contains support for two distinct and even opposed theories of the attribution process. Kelley $(1967,1972)$ has proposed that the perceiver makes attributions after logically weighing information about the covariation of an effect with various possible causes. This covariation information may be observed or assumed (Kelley's, 1972, "causal schema"), but in either case it is processed more or less rationally, in a fashion resembling the analysis of variance (ANOVA). Kelley's model of attribution has come to be known as the ANOVA model for this reason. On the other hand, several recent writers (Pryor \& Kriss, 1977; Taylor \& Fiske, 1975, 1978) have argued that perceivers use a much simpler information-processing strategy: at-

The authors are grateful to Susan T. Fiske, Reid Hastie, and Shelley E. Taylor for their comments on an earlier version of this paper. The research reported here was supported by the University of California Academic Senate.

Requests for reprints should be sent to Eliot $R$. Smith, Department of Sociology, University of California, Riverside, California 92521. tributing causality to the most salient plausible cause instead of weighing many possible causes to make a decision. Salience is used synonymously with Tversky and Kahneman's (1974) use of "availability" to suggest some factor that is literally prominent in the perceiver's field of view (Taylor \& Fiske, 1975) or that is easily retrievable from memory (Pryor \& Kriss, 1977).

A variety of studies have demonstrated that both object salience and the information specified by the ANOVA model can influence perceivers in making causal assignments. Taylor and Fiske (1978) present strong evidence that in many everyday situations perceivers give little thought to issues of causation, making attributions to salient stimuli off the "top of the head." These situations are primarily routine ones that involve the perceiver very little. On the other hand, in psychological experiments where information is available and instructions (or implicit demands) urge the subject to consider evidence carefully before making a response, perceivers can respond to information in the fashion Kelley suggests (McArthur, 1972). 
Is it possible to identify the processes by which both salience and the types of information identified by Kelley influence attributions? That is, going beyond the simple demonstration that different manipulations do influence attributions, research can address the question of how they do. One line of research that may give insights into processes is that aimed at identifying points of demarcation between salience and ANOVA processes (cf. Taylor \& Fiske, 1978, Section VII). One prediction is that salience effects might weaken or vanish, and the more thoughtful ANOVA processes take over, when the attributor is highly involved in the situation about which he or she is making causal judgments. Taylor, Crocker, Fiske, Sprinzen, and Winkler (1979) attempted to moderate salience effects on attributions by a variety of manipulations. In several studies they varied distraction, general arousal, and interest in the event to be attributed-all ways of affecting the subjects' degree of involvement in the situation-but were unable to modify the salience effect strongly. This suggests that involvement in events may not lead to more rational, ANovalike processing of them; on the contrary, such engrossment may sometimes prevent more active processing. One can readily imagine a harried attributor tossing off causal judgments as needed in response to minimal cues. Study 1 of this article attempted to manipulate the subjects' degree of involvement in attributional information processing itself, as distinct from involvement in the situation, to determine whether salience effects are weakened when perceivers are forced to give extensive consideration to many possible causes, that is, under conditions that ought to be ideal for the emergence of the more rational information processing described by Kelley's ANOVA model.

The manipulation of salience used in this study was used and validated by Pryor and Kriss (1977). Their Study 1 showed that varying the order of the person and object in a sentence influenced the salience of each word: Specifically, the first-presented sentence element was identified more quickly in a reaction time task. The more salient word was more available in memory. Their Study
2 then showed that this salience manipulation, applied orthogonally to an information manipulation (sentences varying consensus, distinctiveness, and consistency), did affect attributions in the predicted direction. The more salient sentence element (person or object) attracted more attributions from subjects. It can be argued, however, that Pryor and Kriss's (1977) procedure, in its use of simple closed-ended attribution scales as the dependent measures, encouraged subjects to make quick, relatively unthinking attributions and hence encouraged reliance upon the salience cue. The current study focused upon this possibility. This study also examined recall data that may illuminate the processes that underlie the effects of salience and informational factors on attributions.

The present Study 1 conceptually replicates and extends Pryor and Kriss's (1977) Study 2. Pryor and Kriss presented subjects with 16 sentences of the type "Joe likes the film." In a within-subjects experiment they manipulated salience (by varying the order of presentation of subject and object in the stimulus sentence), information (by including additional sentences that contained consensus, distinctiveness, and consistency information pointing to either the subject or object as causal), and verb form (the verb was "like" or "dislike"). They measured attributions of causality to the subject and object on separate 11-point scales but reported analyses only on the difference between these two attributions. The current study largely followed Pryor and Kriss's procedure, with the addition of a between-subjects manipulation intended to influence strongly the amount of time and attention subjects gave to consideration of several possible causes in answering the attribution dependent measures, and a recall measure, administered after the conclusion of the attribution questionnaire, in which subjects attempted to reproduce the stimulus sentences.

Three specific predictions are made for this study.

1. Salience phenomena have been viewed as characteristic of perceivers who give attributional responses on the basis of a quick, sketchy analysis because they are uninvolved 
-or are overly engrossed and pressed for time. This view implies that increased thought or information processing concerning attributions should diminish the effects of the salience manipulation.

2. If salience effects are mediated by cognitive availability or similar factors that affect memory, then relationships between attributions and memory should appear. Differential availability of the subject and object of the sentence should influence the way the sentence is recalled as well as the attribution that is made. In general, relationships between memory and attribution should provide information about the cognitive dynamics of salience.

3. Finally, the recall measures may be influenced by the manipulations (salience and information) that are applied to the sentences and are known to affect attributions. Effects of salience on recall would confirm once again (as in Pryor \& Kriss's Study 1) that salience operates through an effect on the stimulus sentence's representation in memory (thereby influencing its recall). Effects of covariation information on recall are a more interesting possibility; they would point to a close connection between attribution and the cognitive processes involved in sentence comprehension and recall.

\section{Study 1}

\section{Method}

Overview. The first study replicates Pryor and Kriss's Study 2, extended in three ways. The first extension is the addition of a between-subjects manipulation intended to influence the amount of thought subjects give to their attributions. Subjects in the little thought condition received the closedended dependent measures used in the Pryor and Kriss study, two 11-point scales for attributions to the subject and object of the stimulus sentence (wording was, "To what extent was the given situation caused by [name of person or object] ?"). Subjects in the moderate thought condition were instructed to think of and write in their own words one explanation for the event presented in the stimulus sentence and then to code that explanation using the two scales. Subjects in the extensive thought condition were asked to think of and write several (as many as five) different possible explanations for the given event, to consider them all carefully, to indicate the best explanation and, finally, to code that cause using the two closed-ended scales.
A larger amount of careful and systematic thought should be given to the final closed-ended responses in the moderate and extensive thought conditions than in Pryor and Kriss's little thought condition.

The second extension is the addition of a recall measure. After subjects completed the experimental booklet that presented the 16 sentences and causal measures, they were given a sheet of paper with instructions to "list below as many [sentences] as you can remember-just the sentences, not the additional information that was given." Each sentence was coded for whether it was recalled with subject first or object first (paralleling the salience manipulation) and whether the subject and object of the sentence were each recalled.

The third extension is of a minor nature. Eight different positive verbs and eight different negative verbs were used in place of Pryor and Kriss's two verbs "like" and "dislike." This was intended to provide confirmation of their unexpected finding of a Verb Form $\times$ Information interaction.

Subjects. The subjects were 84 undergraduate and graduate students ${ }^{1}$ (28 randomly assigned to each of the three conditions) at the University of California, Riverside, who were paid for their participation. Subjects were run in groups that were heterogeneous as to experimental condition and in which communication among subjects did not take place. The experimenter who administered the questionnaire and debriefed the subjects was blind to subjects' conditions.

Materials. Sixteen verbs were used in sentences, 8 different verbs implying a positive relationship between the subject and object of the sentence (e.g., likes, agrees with, is pleased with, helps, values), and 8 different negative verbs (e.g., dislikes, despises, complains about, is angry with). With this exception, the salience and information manipulations are as described by Pryor and Kriss (1977). Salience refers to the order of presentation of the subject and object, and information to the consensus, distinctiveness, and consistency factors presented in the same manner as in McArthur (1972). The salience manipulation is not equivalent to active versus passive syntactic forms of the sentence, although it does overlap somewhat. Some sentences (such as the first example below) are syntactically passive in the person-salient version (e.g., "Penny is disgusted by the turnips ...") and active in the object-salient version (as shown). Examples are (object salient with object information):

The turnips at dinner disgust Penny.

The turnips at dinner disgust almost everybody.

Most other vegetables do not disgust Penny.

In the past the turnips at dinner have almost always disgusted Penny.

1 The five graduate-student subjects were all from fields unrelated to social psychology, and exclusion of their data does not materially affect the results. 
(person salient with person information):

Phil complains about his co-worker's habits.

Almost nobody else complains about Phil's coworker's habits.

Phil complains about almost everybody's habits.

In the past Phil has almost always complained about his co-worker's habits.

(object salient with person information):

The vegetables at the Italian restaurant are liked by Tony.

The vegetables at the Italian restaurant are not liked by almost anybody else.

Most other vegetables are liked by Tony.

In the past the vegetables at the Italian restaurant have almost always been liked by Tony.

The salience, information, and verb form (positive/ negative) manipulations were all orthogonal, forming a $2 \times 2 \times 2$ design of which each subject completed two entire replications, for a total of 16 different sentences. Four questionnaire forms were used, so that each stimulus sentence was presented to equal numbers of subjects in each of the four Salience $X$ Information conditions. The order of presentation of the 16 sentences was randomized separately for each subject to avoid systematic order effects. The dependent measures (attribution and recall) were described above.

\section{Results}

The results fall into three sections: attributions, recall, and relations between attribution and recall. Attributions and recall were analyzed in a Thought $\times$ Salience $\times$ Information $\times$ Verb Form $\times$ Replication $(3 \times 2 \times 2$ $\times 2 \times 2$ ) analysis of variance, with all but thought being within-subjects factors. Replication is always treated as a dummy factor, and any significant effects it may have are ignored; it has no significant interactions that qualify effects discussed below. ${ }^{2}$

Attribution measures. The attribution dependent measures were subject attribution, object attribution, and the difference between them (the measure used by Pryor \& Kriss, on which a higher score means more person attribution). In the current data, the withinconditions correlation between subject and object attribution averages $-.470, p<.05$, justifying the use of the difference score. In addition, results for all three measures are quite similar. ${ }^{3}$ Considering the difference measure, four significant effects appeared, the first three of which were also obtained by Pryor and Kriss. ${ }^{4}$ Covariation information strongly affected attributions: The mean attribution score was 5.08 with person-oriented information and -1.74 with object-oriented information. ( $F_{\mathrm{S}}$ are shown in Table 1.) Salience also had a significant effect on attributions. The mean was 2.03 when the sentence was presented with the person salient and 1.32 when the sentence was presented with object salient. A significant Verb Form $\times$ Information interaction also appeared, with covariation information having more impact when the verb was negative than when it was posi-

2 The use of an alternative analysis based on quasi $F$ ratios, recognizing that replication as well as subject is a random factor in the design, does not change the essential conclusions. In the current study, subjects contributed much more variance than replications. The analyses reported here are ordinary $F$ s based on the appropriate interactions with subject as error terms, because this approach is more familiar and because in some analyses to be reported, the use of dichotomous scores renders untrustworthy the high-order interactions required for the computation of quasi $F$ s.

3 The results for the object-attribution dependent measure were the same as for the difference measure, except that the effect of verb type was not significant. The results for the person measure were the same, except that the salience effect was only marginally significant $(p<.06)$ and a three-way Verb Type $X$ Salience $\times$ Information interaction reached significance, indicating that with negative verbs, object information and especially object salience gave rise to much less person attribution. The results for the difference measure thus summarize well the results for the two separate dependent variables: Four of the five significant effects on the difference measure (all except verb type) are at least marginally significant on both the person and object measures.

The strength of the person-object attribution correlation, in contrast to the typical findings of a nonsignificant correlation between measures of situational and dispositional attributions (e.g., Miller, Smith, \& Uleman, Note 1), may be due to the presentation of the informational manipulations. These sentences strongly imply a person or object attribution for each stimulus sentence and hence may sensitize subjects to the idea that person and object attributions are alternatives.

4 Verb form also affected attributions, with more person attributions being given for positive than for negative verbs. The Verb Form $\times$ Replication interaction was significant, however, so the verb form effect should be interpreted with caution (and it receives no further attention in this article). 
Table 1

Analysis of Variance Results for Difference Attribution Measure and Significant Effects in Study 1

\begin{tabular}{lrrrr}
\hline \multicolumn{1}{c}{ Effect } & \multicolumn{1}{c}{$M S$} & $d f$ & $F$ & $p$ \\
\hline Salience & 170.715 & 1 & 6.556 & .013 \\
(Error) Salience $\times$ Unit & 26.038 & 81 & - & - \\
& & & & \\
Information & $15,614.238$ & 1 & 205.811 & .000 \\
Information $\times$ Thought & 435.421 & 2 & 5.739 & .005 \\
(Error) Information $\times$ Unit & 75.867 & 81 & - & - \\
Verb Form $\times$ Information & & & & .001 \\
(Error) Verb Form $\times$ Information $\times$ Unit & 435.435 & 1 & 13.761 & - \\
\hline
\end{tabular}

tive (means appear in Table 2). This replication of Pryor and Kriss's finding on verb form with an extended sample of verbs increases confidence in the effect. This interaction may be due to subjects being more careful (i.e., paying more attention to information) in their attributions for negative events, perhaps because such events have bad implications or are unusual (cf. Kanouse \& Hanson, 1972).

The other attribution effect involved the between-subjects manipulation and so was not part of Pryor and Kriss's findings. The thought manipulation was successful. Subjects in the extensive thought condition wrote down and considered a mean of 3.1 different explanations before selecting the best. Sub-

Table 2

Means of Difference Attribution Measure for Study 1

\begin{tabular}{ccc}
\hline & \multicolumn{2}{c}{ Information } \\
\cline { 2 - 3 } Item & Person & Object \\
\hline Thought $^{\mathrm{a}}$ & & \\
Little & 5.683 & -2.638 \\
Moderate & 5.839 & -1.705 \\
Extensive & 3.723 & -0.862 \\
Verb form & & \\
Positive & & \\
Negative & 4.860 & -0.818 \\
\hline
\end{tabular}

Note. A higher mean indicates more person attribution and less object attribution.

s $n=224$ observations per mean.

b $n=336$ observations per mean. jects in the moderate thought condition wrote down one explanation and then coded it, whereas subjects in the little thought condition had only to make two checkmarks on closed-ended attribution scales. It is notable that the data contain no support for Prediction 1 , which called for the weakening of salience effects in the extensive thought condition; for the Thought $\times$ Salience interaction, $F(2,81)=.78, p=.46$. Salience effects on attributions were as strong when subjects carefully considered several possible explanations as when they simply made two checkmarks on attribution scales. The thought manipulation did interact with information, however, with the impact of covariation information being largest in the little thought condition and smallest in the extensive thought condition. Means appear in Table 2, and $F$ test results in Table 1 .

Recall measures. Four recall measures were analyzed. These were the proportion of sentences recalled with person first, the proportion recalled with object first, ${ }^{5}$ the proportion of persons recalled at all, and the proportion of objects recalled at all. Partial recall was fairly frequent, especially of the form " complains about his co-worker's habits." This example would have been scored as recalled

\footnotetext{
5 These two proportions are independent, as they add to the proportion of sentences recalled at all, and not to 1.0. The order-of-recall variables were scored independently of the initial salience (i.e., order of presentation) of the sentences-that is, any recall of a sentence in a particular order was counted, not just correct recall of order.
} 
with person first, object recalled, person not recalled. The reliability of this coding was near perfect. A second rater checked approximately 30 sentences, with no disagreements on coding.

Both salience and information influenced the order of recall variables. More sentences were recalled with the object first when the object was salient ( $M=.125$ vs. .029), $F(1$, $81)=28.31, p<.001$. A Salience $\times$ Verb Form interaction affected both of the orderof-recall variables. Object salience led to stronger object-first recall, and person salience led to stronger person-first recall, when the verb was negative as compared with positive; for person-first recall, $F(1,81)=4.82$, $p<.05$; for object-first recall, $F(1,81)=$ $5.66, p<.01$.

More important is an interaction of salience with covariation information. Inspection of the Salience $\times$ Information interaction means (Table 3 ) reveals that information has little or no effect on order of recall in the person-salient condition, where nearly all recalled sentences were reproduced accurately with person first-in this condition the mean object-first recall was only .029 . When sentences are presented with object salient, however, there is more variation in the recall measures, and covariation information has an effect. For the Salience $\times$ Information interaction, for person-first recall, $F(1,81)=$ $7.16, p<.05$; for object-first recall, $F(1,81)$ $=9.16, p<.01$. Tests of the simple effects of information within the object-salient condition, using the appropriate pooled error mean square (Winer, 1970, pp. 544-545), reveal that this effect is significant for both the person-first recall and object-first recall measures, $t(81)=3.07, p<.01$, and $t(81)=$ $2.93, p<.01$, respectively. In both cases, covariation information influences recall in the predicted direction, with the factor the information pointed to as the cause more likely to be recalled first. The clear conclusion is that these recall measures suffer respectively from a ceiling effect and a floor effect in the person-salient condition, producing the observed Salience $x$ Information interaction. When the analysis is focused on the object-salient condition in which the absence of ceiling and floor
Table 3

Means for Recall of Verbs by Salience and Information for Study 1

\begin{tabular}{ccc}
\hline & \multicolumn{2}{c}{ Information } \\
\cline { 2 - 3 } Proportion recalled & Person & Object \\
\hline Person salient (person first) & \\
Person first & .426 & .459 \\
Object first & .047 & .012 \\
\hline Object salient (object first) & \\
\hline Person first & .470 & .358 \\
Object first & .098 & .152 \\
\hline
\end{tabular}

Note. Each proportion is based on 336 observations.

effects allows the recall measures to show more variance, the effects of the covariation information manipulation on recall are significant. ${ }^{\circ}$

Correlations between attributions and recall. Finally, we come to the relationships between attributions and recall. These were analyzed as partial correlations to ensure that a true relationship between the variables is present, not a spurious relationship resulting from the variables being affected in similar ways by the manipulated factors (cf. Taylor \& Fiske, 1978, section VI.C). The partial correlations of the two attribution measures (person and object) with the four recall measures were computed across responses, controlling for salience, information, and experimental conditions (thought). This test shows

\footnotetext{
- Other effects on recall measures, not of central theoretical significance, can be briefly mentioned: More sentences were recalled person first, and more persons were recalled, in the extensive thought condition than in the other two conditions, $F(2,81)=$ $4.54, p<.05$. Person recall was influenced strongly by verb form, with more persons presented with positive verbs being recalled (means of .317 vs. .208), $F(1,80)=19.05, p<.001$. The proportion of objects recalled was influenced by salience, with more object recall when the object was salient, $F(1,80)=$ $5.95, p<.05$. Both object recall and person-first order of recall were influenced by a three-way interaction of Thought $\times$ Verb Form $\times$ Salience. However, this effect appears not to be simply inter pretable and does not qualify the interpretation of any other s.gnificant effects, and it will not be discussed further.
} 
that two of the eight relationships are significant. (The probability of obtaining at least two of eight relationships at the .05 level under the null hypothesis is less than .002 by a binomial test.) The proportion of sentences recalled object first was associated with attributions to the object on the object attribution scale, partial $r(1317)=.08, p<.01$, and proportion of persons recalled was associated with higher person attribution on the person scale, partial $r(1317)=.06, p<.05$. Thus, relationships between attribution and recall measures do appear when the experimental manipulations are controlled. The small size of these correlations can be attributed to the facts that (a) the attributional measures may be of low reliability (the two items only correlate .47 ) and (b) these are correlations between a continuous variable and a dichotomy, which can limit the maximum size of correlation coefficients to substantially less than 1 . (Correction of these correlations for the attenuation due to the use of a dichotomy yields estimates of .15 and .08 for their "true" sizes, respectively; Harshbarger, 1977, p. 440.)

\section{Discussion}

Three basic findings were replicated in this study, and two new relationships were uncovered. Salience affects both recall and attribution, and covariation information affects attribution, as Taylor and Fiske (1975) and Pryor and Kriss (1977) have shown. In addition, information affects recall, in the objectsalient condition where the recall measures showed adequate variance. Also, a partial correlation between recall and attributional measures emerged (controlling for the experimental factors).

Contrary to Prediction 1, thought (the extensive thought condition compared to moderate and little thought conditions) did not diminish the effects of the salience manipulation: no Salience $\times$ Thought interaction appeared. This supports the findings by Taylor et al. (1979) that salience effects remain robust despite a wide variety of manipulations. Rather than minimizing the effects of salience, greater thought appears to decrease the importance of the covariation information manipulation. The extensive thought manipulation was specifically intended to influence subjects to consider several explanations and choose the best one, rather than to engage in simple "top of the head" processing of just one causal possibility - the most salient one. The manipulation was evidently successful, as subjects in the extensive thought condition considered a mean of 3.1 different explanations. Yet the impact of salience was not reduced by the manipulation. A couple of points should be made about the thought manipulation. First, the particular manipulation used does not artifactually bias subjects to give more or less weight to any particular kind of information as a determinant of causal attributions (e.g., the covariation information); it asks them only to choose the best cause in the light of all available information. However, the manipulation may have other effects besides the intended one. For example, there may be a motivational effect: In the extensive thought condition subjects may have perceived the task as more important (since they were asked to spend so much time at it) and as a result may have tried harder to be accurate in their attributions. Still, the earlier theory of salience (as based on unthinking top-of-the-head responses) would predict that such a motivational effect would also reduce the impact of salience-contrary to what was found. The thought manipulation thus seems adequate to support the conclusion that we wish to draw from it: Salience effects persist even when subjects give careful consideration to many possible causes of an event.

The fact that salience manipulations have an impact over a broad range of conditions indicates that salience is not just a matter of making attributions without thinking. Instead, salience effects appear to be related to such cognitive processes as the way the stimulus sentence is perceived and encoded in the first place, giving rise to the observed memory-attribution links. These links are both the effects of information on recall (implicating attribution processes in the comprehension and recall of sentences) and the partial correlations between recall and attribution indices. 
A simple model can account for the findings. In carrying out the experimental task, subjects must first comprehend the stimulus materials, then report their attribution, then (in the recall task) report their memory of the sentence itself. Results of Study 1 show that salience and information affect both the attribution and recall results and also that there is a partial correlation between attribution and recall indices. These effects can most parsimoniously be accounted for by the hypothesis that attributional processing and recall both operate from a single internal, encoded representation of the stimulus sentence to which the subject refers in answering the questions he or she is posed. The experimental factors that affect both attribution and recall can thus parsimoniously be assumed to affect the nature of the internal representation and thereby, indirectly, the dependent variables. It is easy to see that salience may affect the way a sentence is encoded, but the hypothesis that covariation information does so as well is novel. It has been generally assumed that such informational manipulations affect attribution but do not affect memory per se (Pryor \& Kriss, 1977; Taylor \& Fiske, 1975). Here, however, information is shown to affect recall, so parsimony suggests that the information factor too may operate through an effect on the representation of the sentence in memory. This proposition will be further tested in Study 2.

The subject's reporting of the attribution could then take place in either or both of two ways. The subject could retrieve from memory an attribution that was made during encoding and report it, or the subject could retrieve a representation of the sentence as well as of the additional consensus, distinctiveness, and consistency information and make a causal judgment at the time of retrieval. This argument that attributions are sometimes made at the time information is stored in memory, rather than subsequently on the basis of data retrieved from memory, has two noteworthy implications. First, as mentioned above, it is contrary to Pryor and Kriss's (1977) interpretation of salience. They raise two arguments against the idea that attributions are encoded and retrieved from memory: Since their subjects were not told that the experiments involved an attribution task they had no reason to make attributions; and covariation information did not influence the speed of recall in their study, as would be expected if attributions were involved in information encoding and storage. In response it can be argued that (a) social psychologists have often claimed that attributions are frequently made by people in their everyday lives, not just when experimenters ask for them. If we did not believe this, there would be little point in studying attributions. (b) Subjects in the Pryor and Kriss study, as well as in the current study, received the covariation informational manipulations with each sentence. Since these strongly point to either the subject or the object as the cause, it would be surprising if reading these stimuli did not generate some causal thinking. (c) In the current study, information was shown to influence two recall measures (not measures used by Pryor \& Kriss).

Second, the notion that attributions can be made as information is stored in memory is consistent with many current theories of sentence comprehension and memory in cognitive psychology that give important places to inferred (attributed) causality. Among others, Kintsch (1974), Norman, Rumelhart, and the LNR Research Group (1975), and Schank (1975) have all proposed theories that involve the representation of "cause" links between propositions. In comprehending a text like "A burning cigarette was carelessly discarded. The fire destroyed many acres of forest," people make the attribution that the cigarette caused the fire and seem to store that inferred causal link as if it were explicitly stated in the text, when they are tested after a 15-minute delay (Kintsch, 1975). The cognitive theories and studies support the idea that attributional (cause-inferring) processing is intrinsically involved in the initial comprehension of sentences and therefore that it goes on all the time, not just when a subject is asked an attributional question. Such theories also imply that the cause generated by the initial attributional processing is represented in memory in some form. Hence relations between recall and causal responses, 
such as those obtained in Study 1, are to be expected.

Our tentative model thus holds that subjects comprehend the stimulus material: They process the sentence as influenced by the salience and information factors and transform it into a representation in memory. Some attributional inferences occur in this process, and causal material is included in the memory representation (Kintsch, 1974, 1975). If subjects are immediately asked to give an attribution, they may report the cause they have stored in memory-but on the other hand, the additional covariation information may still be available to them, so subjects may be able to carry out further attributional processing in response to the question. If a delay intervenes between the study of the material and the attributional question, Kintsch's results suggest that subjects will be more likely to answer the question directly from the memory representation. The additional covariation information will in general have been lost from memory during such a delay. Only the stimulus sentence itself will be retrievable because it was more intensively studied by the subjects and because it is more central; such propositions are better recalled (Kintsch, 1974). The prediction of this model is thus for a stronger relationship of attribution and recall indices after a delay than immediately and for a greater influence of salience on attributions (via its influence on the memory representation) after the delay. These hypotheses parallel Kintsch's (1975) results showing that after a delay causal relations seem to be retrieved as part of a person's encoded memory for the event itself, not as separately encoded information.

Study 2 was conducted as a test of these ideas. In particular, it was predicted that (a) the effect of information on recall found in Study 1 should be replicated within the object-salient conditions where some variation exists in the order-of-recall measures; (b) the partial correlation of attribution and recall variables should be replicated; (c) this attribution-recall relationship should increase with time after the presentation of the sentences, since other information (e.g., the covariation information) should have decayed from memory and the subject will be forced to base his attribution and recall responses on the same encoded memory representation of the sentence (cf. Kintsch, 1975); (d) the effect of salience on attributions should also be stronger after the delay, since that effect is presumed to be mediated by the memory representation.

\section{Study 2}

\section{Method}

Overview. Subjects read sentences similar to those in Study 1, together with the salience and information manipulations, presented as materials for a study of memory. After studying the sentences for 8 minutes, some subjects (randomly assigned to the immediate condition) were asked to recall as many sentences as they could in 10 minutes, writing each one and then answering the two closed-ended subject and object attribution questions about it (essentially Pryor \& Kriss's questions). During this time, the other subjects (those in the delayed condition) performed an irrelevant anagrams task. A 20-minute delay then followed, during which the subjects listened to and took notes on a lecture on an unrelated topic. Finally, the memory and attribution dependent measure was administered to all subjects, again for 10 minutes. Thus, immediate recall subjects filled out the measure beginning only seconds after they had finished studying the sentences, and delayed subjects filled it out 30 minutes later.

Subjects. The 107 subjects were volunteers drawn from introductory social psychology courses at the University of California, Riverside (26 subjects) and at New York University (81 subjects). The study was run during a class period, prior to exposing the students to material concerning attribution in the course.

Materials. The 16 sentences used in this study were written to have a person as subject and a nonhuman object, to eliminate ambiguity in the wording of the attribution questions (see below). Some sentences from the first study were retained and some new ones were written. The salience and information manipulations were as described for Study 1. In this study, to simplify the experimental materials and the task of balancing cell sizes in two locations 3,000 miles apart, sentences were nested within (Salience $X$ Information), so that just one form of stimulus presentation needed to be prepared. That is, each sentence was presented in just one Salience $X$ Information condition.

The dependent measure consisted of 16 occurrences of three questions: "Write down one of the sentences here, as exactly as you can remember it-just the sentence, not the additional information about it" (i.e., the information manipulation), followed by person and object attribution questions. These were the 
same as those used in the little thought condition in Study 1, except that instead of including the person's name and the object from the sentence (which would have constituted a powerful recall cue for each sentence), the questions asked, "To what extent was the event described in the sentence caused by the person named in the sentence" and "caused by the object the person was reacting to."

Coding of variables. From the dependent measure, each stimulus sentence was coded as recalled with person first, with object first, or not recalled; the person was coded as recalled or not; and the object was coded as recalled or not. Four recall measures identical to those in Study 1 result. Since attribution measures are available in this design only for sentences that were recalled, analyses involving the attribution variables must exclude sentences that were not recalled. For such analyses the two order-ofrecall variables are redundant (since a recalled sentence must be scored as either recalled person first or recalled object first) and only one can be used. The person and object attribution scales were used in their original form, with a higher score meaning in each case less person or object attribution. A difference score was not used in this study, since the correlation of the two attribution scales was only -.26 , not strong enough to justify using the difference as a single summary measure.

Analysis. Attributions cannot be analyzed in a repeated-measures design in this study because they are not given for sentences the subject did not recall. Recall, however, can be so analyzed. The anova design is Time $\times$ Sentence Nested Within (salience by information), the latter three all being within-subjects variables. Attributions and the relationships among recall and attribution variables can be analyzed with the sentence as the unit of analysis. Since a subject typically recalled several sentences, the observations in this mode of analysis are not independent of each other, giving rise to some degree of bias. The bias is small, however, since the variance due to sentences is generally many times greater than that due to subjects in these analyses, and this analysis is the most effective available for examining the questions of interest. ${ }^{7}$

\section{Results}

It was predicted first that this study would replicate an effect found in Study 1, an impact of information on the order of recall of a sentence, within the object-salient condition. Unfortunately, no such effect was found, and the reason became clear in examining the ANOVA table: The design used, with sentences nested within information, had very low power to detect such effects, since the sentence factor contributed much variance (sentence was significant at the .001 level for both the order-of-recall dependent measures). This indicates that the design should have crossed Sentence $\times$ Information Condition, as Study 1 did, yielding higher power for tests of information effects (cf. Kenny \& Smith, Note 2).

The second prediction was for a withinconditions relationship between attribution and recall. This was examined by computing correlations between the three recall variables (order of recall, person recalled, object recalled) and the two attribution variables (person and object attributions) separately for the four combinations of Time $\times$ Salience. (Information need not be considered, since it did not significantly affect recall; thus, it cannot generate spurious correlations between recall and attribution measures.) One of the 12 correlations from the immediate subjects was significant: In the object-salient condition, object recall was associated with less person attribution, $r=.14, p<.05$. For the delayed subjects, 4 of 12 correlations reached the .05 level, all in the object-salient condition. All three recall variables (object-first order of recall, person recall, and object recall) were associated with more person attribution $(r=$ $-.16,-.22$, and -.17 , respectively). Object recall was also associated with more object attribution, $r=-.20$. Overall, obtaining 5 of 24 correlations at the .05 level by chance

\footnotetext{
7 The use of analyses that may contain technical violations of assumptions has precedents in the attribution literature. McArthur (1972) found herself in a situation similar to ours, argued that the bias due to ignoring subject effects would be "negligible," and proceeded with an analysis that is conceptually similar to the ones we perform. Our data permit a reassurance like the one she was able to give: The correlation of recall responses given by the same subject is negligible relative to item effects. In addition, we have conducted reanalyses to test the effects of the violations of assumptions. Removal of subject effects from the attribution variables (by subtracting each subject's mean score from each observation) and reanalyses of these corrected scores show that the major points of the paper are unaffected by the nonindependence in the original data. This is the case with the partial correlations between memory and attribution in Studies 1 and 2, the ANOva on attribution in Study 2, and the path models in Study 2. (The other analyses reported in the paper explicitly incorporate the subject variable and so are not subject to the criticism of nonindependence of observations.)
} 
alone has a binomial probability of less than .002 : a clear demonstration of relationships between recall and attributions.

The third prediction was that the relationship between recall and attributions should be stronger when recall was delayed. This issue can be illuminated by path diagrams showing the influences of the experimentally manipulated factors on recall and attribution variables, separately for the immediate and delayed recall conditions. Using the conventions of path analysis to draw the diagrams (Duncan, 1975), the following are the results for the most sensitive recall and attribution variables, object-first recall and person attribution. ${ }^{8}$

In Figure 1, the straight arrows represent the causal effects (numerically, the standardized regression coefficients) of the manipulated variables on the two response variables. The residual causes $u$ and $v$ represent all other causes of the responses (i.e., error variance). The curved arrow between $u$ and $v$ represents a correlation between those residual causes, generated theoretically by an unmeasured factor that has a causal impact on both recall and attribution (in this case, the factor is presumed to be aspects of the memory representation of the sentence). Note that the model shows neither the recall nor attribution dependent variables as causing the other. This is a consequence of the theoretical viewpoint sketched above: The recall and attribution processes must work from the same encoded representation of the sentence (so one can expect to find a partial correlation between them, represented in the diagram by the correlation between the residual causes $u$ and $v$ ). However, neither can be presumed to cause the other, even though the recall dependent measure comes first in time-attributions (those made during the initial processing of the sentence) may influence recall as well as the other way around.

In the immediate condition, there seems to be a separation of the recall and attribution processes: Salience affects recall, and the informational manipulation affects attribution. Furthermore, the residual correlation between recall and attribution is nonsignificant. However, the delayed condition shows a different
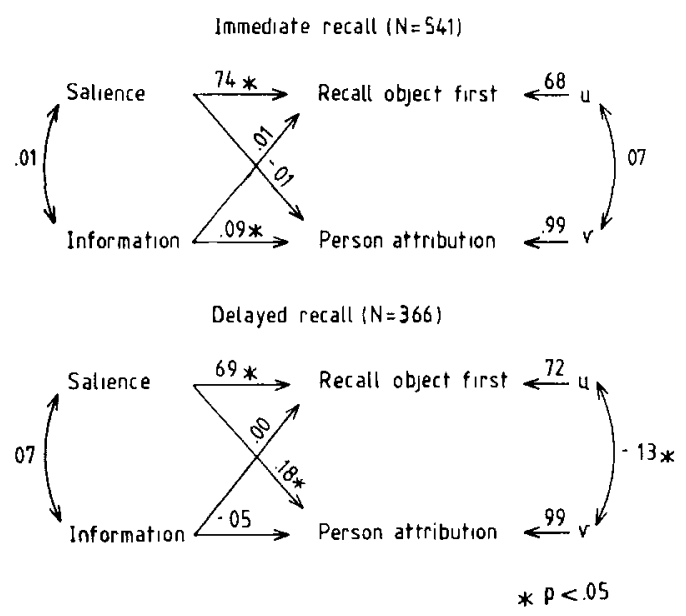

Figure 1. Path diagrams showing relations among manipulated factors, recall, and attribution in immediate and delayed conditions, Study $2 .(p<.05$. $)$

pattern of effects. Salience still strongly affects order of recall, but it now also affects attribution. The information effect has vanished. Finally, the partial (residual) correlation is now significant. (These three correlations or path coefficients differ significantly between the immediate and delayed conditions.) An

8 Detailed results for the other combinations of variables are not presented here for reasons of space. Briefly, the object attribution measure never shows significant relations in these analyses, so only the person scale need be considered. There are three nonredundant recall measures, object-first recall, person recall, and object recall. (Since only sentences that are recalled have attributions, results using the person-first order-of-recall variable are identical to those with the object-first variable except for having the opposite sign.) The major conclusions are the following: (a) For two of the three analyses (objectfirst recall and person recall), the delayed recallattributional correlation is significant and significantly different from the immediate one; (b) in all three cases, there is a significant delayed effect of salience on attribution, significantly greater than the immediate effect; (c) for object recall only, there is a significant delayed effect of information on recallsuch that information implying that the object is causal leads (after the delay) to a greater likelihood of recalling the object. This effect supports our modeI but is not discussed further, since it appears with only one of the three measures. Researchers on salience, at least since Taylor and Fiske (1975), have often obtained theoretically expected effects with only a subset of dependent measures; the exact form of the most appropriate measure is a matter for further study. 
analysis of variance on the person attribution variable verifies that the impact of salience is stronger after the delay: Salience $\times$ Time interaction, $F(1,12)=8.39, p<.05$.

\section{Discussion}

This pattern of findings lends support to the idea that after a delay (though not immediately after study of the sentences) recall and attribution are based on a single encoded representation of the sentence. The effect of salience on that encoded representation seems to be stronger than the effect of the information manipulation: the effect of salience on attribution was significantly stronger after the delay than before. On the other hand, the impact of information was significantly weaker (and nonsignificant) after the delay. This pattern may be accounted for by the salience manipulation's status as part of the sentence itself, whereas the covariation information was presented as separate sentences, subject to memory decay during the 30-minute delay.

Several puzzles remain in the results. First, the residual correlation between recall order and attribution in the delayed condition is in the wrong direction. That is, order of recall has a relation to attributions opposite to that of the order of presentation of the sentence. The reason for this is unknown; the exact form of the representations of sentences in memory is a matter of some controversy (cognitive theories differ widely on the issue). Similarly, we do not yet know what specific characteristics of the representation are responsible for observed differences in order of recall or attribution. Salience does appear to affect the memory representation, but the development of sensitive and relatively pure measures of the affected characteristics remains a topic for future research. Second, the recall-attribution partial correlations in Study 1, although significantly different from zero, were small. This may also be due to the use of measures that are only indirectly related to the true variables of interest, or the effects may actually be relatively small in magnitude (and thus difficult to study). In either case, the elucidation of the relationships among salience, attributions, and the cognitive processes and representations involved in sentence comprehension is a goal that deserves the further research efforts that will be required.

\section{Conclusions}

In summary, this article has attempted to accomplish three things. It has presented evidence that one effect of salience-the dependence of attributions upon the order of presentation of person and object in a stimulus sentence-is surprisingly robust. The effect holds up even when the subject is instructed to invent several possible causes and pick the best one and becomes stronger, rather than weaker, after a delay that forces subjects to rely more on an encoded memory. This finding led to the idea that salience processing involves more than just top-of-the-head responses but may reflect the basic cognitive processes underlying sentence comprehension and the storage of events in memory. (This analysis could be extended to visual manipulations of salience, as in Taylor \& Fiske, 1975, by assuming a verbal representation of encoded visual material; cf. Chase, 1978.) One implication of the data is that strict lines of demarcation between salience processes and more thoughtful attribution processes do not exist. Rather, one must seek to understand how salience and informational factors exert influence on the perceiver across a series of stages of encoding, processing, and recall.

Second, two new findings concerning memory are presented (in addition to the replication and extension of Pryor \& Kriss's finding that salience affects memory as well as attributions): an effect of information on recall (in Study 1) and a relationship between attributions and characteristics of the recall of a sentence (in both studies). Further research is clearly needed to investigate the effects of different types of stimuli and different delay periods and particularly to identify the most appropriate and sensitive recall measures with which to elucidate the cognitive processes underlying attribution. The present findings point to a close relationship between the processes that subjects use in reporting attri- 
butions and in reporting recall; in particular, we advance the hypothesis that both of these processes work from an encoded representation of the stimulus that the subject initially forms. This interpretation is supported by the fact that both the recall-attribution relationships and the effects of salience are increased by a delay (Study 2). Although the support of the hypothesis by these findings is inferential, theory and data from the literature on text comprehension and representation, cited earlier, also argue strongly for such an encoding process, which can involve attributional processing at the time of the encoding and storage of a stimulus as well as at the time of its retrieval. Thus attribution can mediate memory, as well as memory mediating attribution.

Third, an implicit message of these arguments has been that the formulation of explicit theories of information processing in an experimental task can be a productive approach for future research. Instead of asking about whether one variable mediates another in the sense that a statistical relationship between them can be established, we should attempt to understand how the subject goes about performing the task and therefore how the results of a process such as encoding or memory can influence other subsequent processes. Such explicit theories of stages in information processing lend themselves readily to test by means that have been used in cognitive psychology with great success for the past several years (Schneider \& Shiffrin, 1977; Shiffrin \& Schneider, 1977; Taylor, 1976).

\section{Reference Notes}

1. Miller, F. D., Smith, E. R., \& Uleman, J. Can we measure causal attributions? Manuscript submitted for publication, 1978.

2. Kenny, D. A., \& Smith, E. R. A note on the analysis of designs in which subjects receive stimuli only once. Manuscript submitted for publication, 1978.

\section{References}

Chase, W. F. Elementary information processes. In W. K. Estes (Ed.), Handbook of learning and cognitive processes: Vol. 5. Human information processing. Hillsdale, N.J.: Erlbaum, 1978.
Duncan, O. D. Introduction to structural equation models. New York: Academic Press, 1975.

Harshbarger, T. Introductory statistics: $A$ decision map approach. New York: Macmillan, 1977.

Kanouse, D. E., \& Hanson, L. R. Negativity in evaluations. In E. E. Jones, D. E. Kanouse, H, H. Kelley, R E. Nisbett, S. Valins, \& B. Weiner (Eds.), Attribution: Perceiving the causes of behavior. Morristown, N.J.: General Learning, 1972.

Kelley, H. H. Attribution theory in social psychology. In D. Levine (Ed.), Nebraska Symposium on Motivation (Vol, 15). Lincoln: University of Nebraska Press, 1967.

Kelley, H. H. Causal schemata and the attribution process. In E. E. Jones et al. (Eds.), Attribution: Perceiving the causes of behavior. Morristown, N.J.: General Learning Press, 1972.

Kintsch, W. The representation of meaning in memory. Hillsdale, N.J.: Erlbaum, 1974.

Kintsch, W. Memory representations of text. In R. L. Solso (Ed.), Information processing and cognition. Hillsdale, N.J.: Erlbaum, 1975.

McArthur, L. The how and what of why: Some determinants and consequences of causal attribution. Journal of Personality and Social Psychology, 1972, 22, 171-193.

Norman, D. A., Rumelhart, D. E., \& the LNR Research Group. Explorations in cognition. San Francisco: Freeman, 1975.

Pryor, J. B., \& Kriss, M. The cognitive dynamics of salience in the attribution process. Journal of Personality and Social Psychology, 1977, 35, 49-55.

Schank, R. Conceptual information processing. Amsterdam: Elsevier, 1975.

Schneider, W., \& Shiffrin, R. M. Controlled and automatic human information processing: I. Detection, search, and attention. Psychological Review, $1977,84,1-66$.

Shiffrin, R. M., \& Schneider, W. Controlled and automatic human information processing: II. Perceptual learning, automatic attending, and a general theory. Psychological Review, 1977, 84, 127-190.

Taylor, D. A. Stage analysis of reaction time. Psychological Bulletin, 1976, 83, 161-191.

Taylor, S. E., Crocker, J., Fiske, S., Sprinzen, M., \& Winkler, J. The generalizability of salience effects. Journal of Personality and Social Psychology, 1979, 37, 356-367.

Taylor, S. E., \& Fiske, S. T. Point of view and perceptions of causality. Journal of Personality and Social Psychology, 1975, 32, 439-445.

Taylor, S, E., \& Fiske, S. T. Salience, attention, and attribution: Top of the head phenomena. In L. Berkowitz (Ed.), Advances in experimental social psychology (Vol, 11). New York: Academic Press, 1978.

Tversky, A., \& Kahneman, D. Judgment under uncertainty: Heuristics and biases. Science, 1974, 185, 1124-1131.

Winer, B. J. Statistical principles in experimental design. New York: McGraw-Hill, 1970.

Received December 11, 1978 